\title{
MÉTODOS DE PESQUISA OPERACIONAL NA DEFINIÇÃO DE METAS PARA INDICADORES DE SUSTENTABILIDADE EM UMA EMPRESA DO SETOR ELÉTRICO BRASILEIRO NO CONTEXTO DA AGENDA 2030
}

\author{
Katia Cristina Garcia \\ Cepel - Centro de Pesquisas de Energia Elétrica \\ Av. Horácio Macedo, 354 - Cidade Universitária, Ilha do Fundão - Rio de Janeiro - RJ \\ garciak@cepel.br \\ Rodrigo Gomes Távora Maia \\ UFRJ - Universidade Federal do Rio de Janeiro \\ Av. Athos da Silveira Ramos, 149 - Ilha do Fundão - Rio de Janeiro - RJ \\ rgtmaia@gmail.com \\ José Francisco Moreira Pessanha \\ Cepel - Centro de Pesquisas de Energia Elétrica \\ Av. Horácio Macedo, 354 - Cidade Universitária, Ilha do Fundão - Rio de Janeiro - RJ \\ francisc@cepel.br
}

\section{RESUMO}

O trabalho discute o uso de métodos estatísticos de previsão e o uso da análise envoltória de dados como auxílio na definição de metas para indicadores de sustentabilidade no contexto da Agenda 2030. A metodologia proposta consiste no tratamento das séries temporais com a identificação e remoção de outliers a partir da aplicação do método Loess, na realização das previsões de acordo com os modelos de suavização exponencial, na definição do benchmark a partir da Análise Envoltória de Dados (DEA) e posterior definição da meta global e, por último, na distribuição da meta pelo método de mínimos quadrados aliando o benchmark com as previsões. A metodologia foi aplicada em uma empresa do setor elétrico brasileiro para os indicadores de consumo de energia elétrica e consumo de água da rede de abastecimento das atividades administrativas.

Palavra-chave: Sustentabilidade Empresarial; Métodos de Previsão; Análise Envoltória de Dados; Indicadores de Sustentabilidade; Agenda 2030; Objetivos de Desenvolvimento Sustentável.

\begin{abstract}
The present paper discusses the use of statistics methods of forecasting and the use of data envelopment analysis to assist in the definition of goals for sustainability index in the context of Agenda 2030. The proposed methodology consists in the time series analysis using Loess method for identification and removal of outliers, forecasting the time series using exponential smoothing, in the definition of the benchmark using the Data Envelopment Analysis (DEA) and posterior definition of global goal and finally, in the distribution of global goal by the least squares method, combining the benchmark and the forecasting. For
\end{abstract}


practical example, this methodology was applied to a company of the Brazilian electric sector, using the electric energy consumption index and the water consumption index from administrative activities.

Keywords: Corporate Sustainability; Prediction Models; Data Envelopment Analysis (DEA); Sustainability Index; Agenda 2030; Sustainable Development Goals.

\section{Como Citar:}

GARCIA, Katia Cristina; MAIA, Rodrigo Gomes Távora; PESSANHA, José Francisco Moreira. Métodos de Pesquisa Operacional na Definição de Metas para Indicadores de Sustentabilidade em uma Empresa do Setor Elétrico Brasileiro no Contexto da Agenda 2030. In: SIMPÓSIO DE PESQUISA OPERACIONAL E LOGÍSTICA DA MARINHA, 19., 2019, Rio de Janeiro, RJ. Anais [...]. Rio de Janeiro: Centro de Análises de Sistemas Navais, 2019. 


\section{INTRODUÇÃO}

Ao final de 2015, como uma continuação e ampliação dos chamados Objetivos de Desenvolvimento do Milênio (ODM), foram lançados os Objetivos de Desenvolvimento Sustentável (ODS), durante a Cúpula das Nações Unidas para o Desenvolvimento Sustentável, em Nova York. As negociações começaram em 2013, seguindo mandato emanado pelo país sede da Conferência Rio+20, o Brasil. Na prática, os chamados ODS serão responsáveis por orientar as políticas públicas e as atividades de cooperação internacional nos próximos anos, compondo a chamada Agenda 2030, com indicativos do "futuro que queremos”.

Diferente dos ODM, os ODS são aplicáveis a todas as nações do mundo, e mais do que isso, não apenas pelos governos, mas também com responsabilidades compartilhadas com a indústria, empresas privadas e sociedade. Pode-se arriscar dizer que o papel das empresas é chave para o alcance dos ODS, principalmente nos países onde os recursos financeiros são limitados.

Considerando a importância estratégica do setor elétrico para o alcance de vários dos ODS como, por exemplo, o ODS 7 (Energia Limpa e Sustentável) e o ODS 13 (Ação contra a Mudança Global do Clima), discutir a contribuição do setor para a Agenda 2030 torna-se imperativo.

As empresas do setor vêm de uma forma geral, trabalhando a questão da Sustentabilidade Empresarial de maneira crescente nos últimos dez anos. A internalização dos conceitos de Desenvolvimento Sustentável e do triple bottom line (ELKINGTON, 1994 apud SARTORI, 2016, p. 37), a identificação das questões de sustentabilidade materiais no contexto da empresa, o mapeamento de possíveis ações para incremento da sustentabilidade ambiental, econômica e social, a definição de indicadores de sustentabilidade para monitoramento das ações e a comunicação às partes interessadas são algumas das etapas que exemplificam esta busca das empresas.

Porém, no novo contexto mundial, estas etapas não são mais suficientes para garantir uma boa gestão em sustentabilidade. Os ODS apontam objetivos específicos que precisam ser monitorados com indicadores a partir da definição de metas claras, que viabilizem o atendimento até o ano de 2030. Esta necessidade impõe às empresas o estudo de metodologias que auxiliem na definição de metas, de forma a atender às demandas externas e internas dos diferentes stakeholders, ao mesmo tempo em que vislumbram o atendimento aos ODS no que tange a responsabilidade assumida frente ao seu país. Ainda que esse movimento ocorra de forma voluntária, o mercado passa a exigir um posicionamento formal das empresas, como uma forma de diferenciação. Uma prova disto é que ações e indicadores para monitoramento dos ODS já estão sendo apresentados nos Relatórios de Sustentabilidade de diversas empresas do setor nos últimos dois anos, normalmente seguindo a metodologia SDG Compass (CEBDS, 2018).

Esta metodologia foi elaborada pela GRI (Global Reporting Initiative) em conjunto com Pacto Global, WBCSD (World Business Council for Sustainable Development) e a ONU, a fim de orientar a ação das empresas para implantação dos ODS em suas estratégias de negócios. A metodologia tem cinco passos. O primeiro passo definido pela metodologia é o mapeamento dos impactos atuais, potenciais, positivos e negativos de suas atividades nos ODS, em toda cadeia de valor. O segundo passo é selecionar os indicadores e definir uma forma de coleta. No passo 3, são estabelecidas prioridades para planos de ação e melhoria, com estabelecimento de metas. Para isto deve-se selecionar os indicadores ou KPIs (key performance indicators) adequados de forma a garantir uma boa condução, monitoramento e comunicação dos resultados. No passo 4 , as metas de sustentabilidade são integradas às estratégias de negócio, e no passo 5 , todo o processo e progresso deve ser relatado e 
comunicado às partes interessadas.

O presente artigo discute o uso de métodos de pesquisa operacional em uma metodologia para definição de metas de sustentabilidade no contexto da Agenda 2030 para uma empresa holding e suas unidades de negócios. Mais especificamente, a metodologia proposta baseia-se no emprego de métodos de previsão de séries temporais e na Análise Envoltória de Dados (Data Envelopment Analysis - DEA) para a definição de metas para os consumos de água e energia na holding e suas unidades de negócios.

A utilização de métodos de previsão é útil na definição de metas factíveis, mas que consigam impulsionar planos de ação que garantam uma contribuição efetiva da empresa para o alcance dos ODS. Assim, as previsões do consumo de energia e água para a holding são obtidas a partir da soma das previsões calculadas separadamente para cada uma de suas unidades de negócios, portanto, uma abordagem bottom-up. Já a definição das metas para os consumos de água e energia segue uma estratégia top-down, em que, inicialmente definem-se metas globais para a empresa holding por meio de modelos DEA e, na sequência, as metas globais são decompostas em metas locais para cada unidade de negócio com o auxílio de um modelo de mínimos quadrados restritos, cujas restrições incluem os limites mínimos e máximos para as metas locais em cada unidade de negócio, limites definidos pelos intervalos de previsão, e uma restrição de compatibilização das metas locais com a meta global para a holding.

Este trabalho apresenta, além da discussão teórica, um caso prático, construído a partir de uma análise com base em séries mensais históricas de diferentes indicadores de sustentabilidade ambiental, como por exemplo, indicadores de consumo de energia elétrica e de consumo de água, que vem sendo utilizados por mais de uma década por uma empresa do Setor Elétrico Brasileiro no monitoramento de seu desempenho em sustentabilidade.

\section{METODOLOGIA PROPOSTA}

A aplicação da metodologia proposta inicia-se com a obtenção de séries temporais mensais de consumo de recursos, e.g., água a energia, coletadas em uma empresa. Conforme ilustrado na Figura 1, a metodologia possui três etapas: tratamento de dados, previsão e benchmarking.

Séries temporais

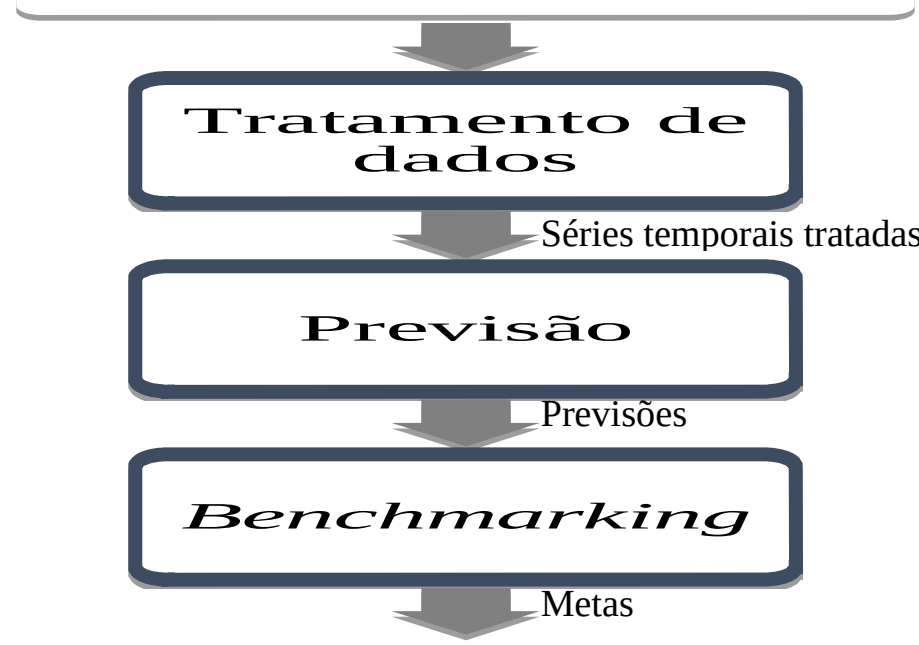

Figura 1. Estrutura da metodologia proposta 


\subsection{Etapa 1: Tratamento de Dados}

As séries temporais podem conter outliers, i.e., dados discrepantes das demais observações. Os outliers são provocados por erros de preenchimentos dos formulários de dados ou por externalidades, e.g., vazamento de água e acidentes. A presença de outliers compromete a qualidade dos modelos de previsão e, portanto, a especificação dos modelos de previsão deve ser precedida por uma etapa de tratamento de dados.

Na metodologia proposta, o tratamento das séries temporais é realizado com o auxílio do método de regressão não paramétrica Loess (CLEVELAND, 1979 apud MARQUETTI; VIALI, 2004, p. 254), por ser um método de tratamento automático, facilitando a sua aplicação para várias empresas.

O método Loess fornece versões suavizadas das séries temporais, acompanhadas de intervalos de confiança. O tratamento de dados consiste em identificar, em cada série analisada, as observações localizadas fora dos limites dos intervalos de confiança e a substituição das mesmas pelos respectivos valores suavizados. Ao final, as séries temporais tratadas estão livres de outliers e podem ser utilizadas no ajuste dos modelos de previsão.

\subsection{Etapa 2: Previsão}

A previsão de séries temporais baseia-se na premissa segundo a qual os fatores que influenciaram o comportamento dos dados no passado continuam influenciando seus movimentos futuros. Assim, analisando o comportamento passado da série temporal podem ser obtidos os elementos para prever o seu comportamento futuro. O propósito dos métodos de previsão é distinguir o padrão de evolução da série (o sinal) de qualquer ruído que possa estar contido nas observações e então usar este padrão (o sinal) para prever valores futuros da série. Conforme ilustrado na Figura 2, uma série temporal pode ser decomposta em três componentes não observáveis: tendência (trend), sazonalidade (seasonal) e irregular (remainder).

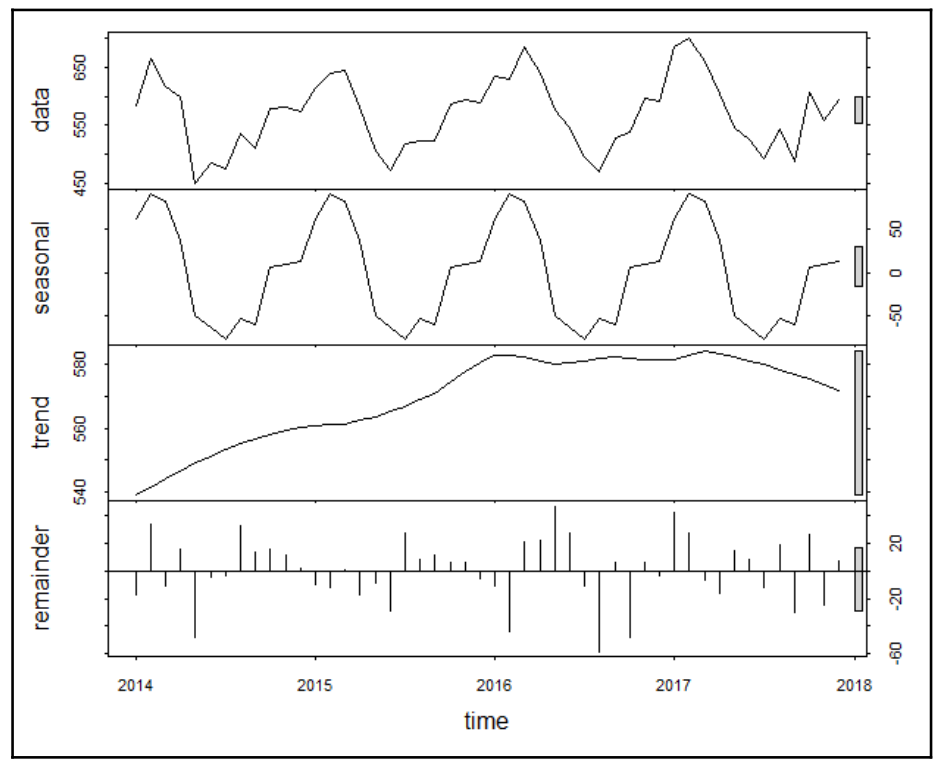

Figura 2. Decomposição de uma série temporal

A componente de tendência aponta para movimentos de longo prazo que indicam o crescimento ou decrescimento da série temporal. Já a componente sazonal descreve as variações periódicas da série temporal com períodos que duram até um ano, como por exemplo, as estações do ano. Por fim, a componente irregular captura as variações de curta 
duração decorrentes, por exemplo, de greves e outros eventos não gerenciáveis. A combinação das componentes supracitadas pode seguir o modelo aditivo, em que a amplitude da variação sazonal é constante ao longo do tempo, i.e., é realizada a soma das componentes, ou o modelo multiplicativo, i.e., a amplitude da variação sazonal aumenta ou diminui como função do tempo, sendo realizada a multiplicação das componentes.

Os métodos escolhidos para a realização da previsão na metodologia foram os métodos clássicos de previsão (MUN, 2010 apud BERTOLO, 2013, p. 5), i.e., os métodos de alisamento exponencial, pelo fato de obter melhores resultados se comparados a outros métodos (MAKRIDAKIS et al., 2018) em séries mensais. Outro fator que auxiliou na escolha desses métodos foi a praticidade de aplicação, já que para o presente estudo foram previstas várias séries temporais.

As componentes tendência, sazonalidade e erro podem ser combinadas de diferentes maneiras, originando um modelo de previsão diferente para cada combinação, em que cada componente compõe parte do acrônimo dado a cada modelo, conforme indicado pelos 19 modelos na Tabela 1.

Tabela 1. Modelos de alisamento exponencial

\begin{tabular}{|c|c|c|c|c|c|}
\hline & \multirow[b]{2}{*}{ Tendência } & \multicolumn{3}{|c|}{ Sazonalidade } \\
\hline & & & Sem & $\begin{array}{c}\text { Aditiv } \\
\text { a }\end{array}$ & Multiplicativa \\
\hline \multirow{5}{*}{$\begin{array}{c}\text { Err } \\
0\end{array}$} & $\begin{array}{c}\text { Aditivo } \\
\text { Multiplicativo }\end{array}$ & Sem & $\begin{array}{l}\text { ANN } \\
\text { MNN }\end{array}$ & $\begin{array}{l}\text { ANA } \\
\text { MNA }\end{array}$ & $\begin{array}{c}- \\
\text { MNM }\end{array}$ \\
\hline & $\begin{array}{c}\text { Aditivo } \\
\text { Multiplicativo }\end{array}$ & Aditiva & $\begin{array}{l}\text { AAN } \\
\text { MAN }\end{array}$ & $\begin{array}{l}\text { AAA } \\
\text { MAA }\end{array}$ & MAM \\
\hline & $\begin{array}{c}\text { Aditivo } \\
\text { Multiplicativo }\end{array}$ & Aditiva Amortecida & $\begin{array}{l}\text { AAdN } \\
\text { MAdN }\end{array}$ & $\begin{array}{l}\text { AAdA } \\
\text { MAdA }\end{array}$ & $\begin{array}{c}- \\
\text { MAdM }\end{array}$ \\
\hline & $\begin{array}{c}\text { Aditivo } \\
\text { Multiplicativo }\end{array}$ & Multiplicativa & $\begin{array}{c}- \\
\text { MMN }\end{array}$ & - & $\begin{array}{c}- \\
\text { MMM }\end{array}$ \\
\hline & $\begin{array}{c}\text { Aditivo } \\
\text { Multiplicativo }\end{array}$ & Multiplicativa Amortecida & $\begin{array}{c}- \\
\mathrm{MMdN}\end{array}$ & - & $\begin{array}{c}- \\
\text { MMdM }\end{array}$ \\
\hline
\end{tabular}

Nos acrônimos apresentados na Tabela 1, a componente erro fornece a primeira letra do nome do modelo, podendo ser "A" (erro aditivo) ou "M" (erro multiplicativo). Já a componente tendência fornece a segunda e, em alguns casos, a terceira letra do nome do modelo, podendo ser " $N$ " (sem tendência), “A" (tendência aditiva), "Ad" (tendência aditiva amortecida), "M" (tendência multiplicativa) ou "Md" (tendência multiplicativa amortecida). A componente sazonalidade fornece a última letra, podendo ser " $N$ " (sem sazonalidade), " $A$ " (sazonalidade aditiva) ou "M" (tendência multiplicativa).

Há algumas restrições de combinações de componentes, por exemplo, uma tendência ou sazonalidade multiplicativa não pode ter o erro aditivo. Adicionalmente vale citar a presença de alguns métodos amplamente conhecidos e aplicados no estudo de previsões de séries temporais como o Holt-Winters Aditivo (AAA e MAA), Holt-Winters Multiplicativo (MAM) e Holt (AAN e MAN).

\subsection{ETAPA 3: BENCHMARKING}

A última etapa consiste na definição das metas globais para a holding, a partir do benchmarking realizado pela Análise Envoltória de Dados (DEA), e na desagregação das metas globais entre as unidades de negócio da holding por meio do método dos mínimos 
quadrados, tendo como restrição os limites inferior e superior dos intervalos de previsão para cada mês do horizonte de estudo (o uso de metas mensais permite monitorar melhor o desempenho das unidades).

Introduzida por Charnes, Cooper e Rhodes (1978), a DEA é uma técnica nãoparamétrica utilizada na avaliação da eficiência de unidades produtivas (Decision Making Units - DMU) comparáveis, i.e., unidades que empregam processos tecnológicos semelhantes na transformação de múltiplos insumos (inputs) em múltiplos produtos (outputs). A abordagem DEA utiliza programação linear para construir uma fronteira de produção, a partir das observações das quantidades de insumos e produtos das DMU avaliadas, sem a necessidade de conhecer a priori qualquer relação de importância (pesos) entre as variáveis consideradas. A fronteira de produção funciona como um benchmark contra o qual podem ser comparados os desempenhos das DMU, as DMU tecnicamente eficientes (eficiência igual a 1) localizam-se na fronteira de produção, enquanto as DMU ineficientes localizam-se abaixo da fronteira. Os desvios das DMU em relação a fronteira quantificam as respectivas ineficiências. Portanto, a fronteira de produção pode ser usada como referência para o estabelecimento de metas para cada DMU.

Há dois modelos DEA clássicos: CCR ou CRS (Constant Return of Scale), proposto por Charnes, Cooper e Rhodes (1978) e BCC ou VRS (Variable Return of Scale), proposto por Banker, Charnes e Cooper (1984). Ambos podem ser orientados ao insumo, caso o objetivo seja fornecer metas para a conservação de recursos (uso eficiente dos insumos), ou orientados ao produto, caso o produto seja definir metas para expansão da produção.

Os modelos CCR (CRS) na Tabela 2 admitem retornos constantes de escala, i.e., qualquer variação nos insumos leva a uma variação proporcional nos produtos. Os modelos CRS fornecem uma medida global da eficiência técnica.

Tabela 2. Modelos CCR (CRS) (adaptado de CASA NOVA; SANTOS, 2008)

\begin{tabular}{|c|c|}
\hline $\begin{array}{l}\text { Modelo CCR (CRS) - Orientado ao } \\
\text { Insumo }\end{array}$ & Modelo CCR (CRS) - Orientado ao Produto \\
\hline $\begin{array}{l}\text { Maximizar } h_{k} \text { (Eficiência) } \\
h_{k}=\sum_{r=1}^{m} u_{r} * y_{r k}\end{array}$ & $\begin{array}{l}\text { Minimizar } h_{k} \text { (Eficiência) } \\
h_{k}=\sum_{i=1}^{n} v_{i} * x_{i k}\end{array}$ \\
\hline $\begin{array}{l}\text { Com as restrições: } \\
\text { 1. } \sum_{r=1}^{m} u_{r} * y_{r j}-\sum_{i=1}^{n} v_{i} * x_{i j} \leq 0 \\
\text { 2. } \sum_{i=1}^{n} v_{i} * x_{i k}=1 \\
\text { 3. } u_{r}, v_{i} \geq 0\end{array}$ & $\begin{array}{l}\text { Com as restrições: } \\
\text { 1. } \sum_{r=1}^{m} u_{r} * y_{r j}-\sum_{i=1}^{n} v_{i} * x_{i j} \leq 0 \\
\text { 2. } \sum_{r=1}^{m} u_{r} * y_{r k}=1 \\
\text { 3. } u_{r}, v_{i} \geq 0\end{array}$ \\
\hline $\begin{array}{l}\text { Sendo: } \\
y_{r}=\text { quantidade de produtor; } \\
x_{i}=\text { quantidade de insumo } i ; \\
u, v=\text { pesos de produtos e insumos; e } \\
r=1 \text { até } \mathrm{m} ; i=1 \text { até } \mathrm{n} ; j=1 \text { até } \mathrm{N}\end{array}$ & $\begin{array}{l}\text { Sendo: } \\
y_{r}=\text { quantidade de produtor; } \\
x_{i}=\text { quantidade de insumo } i ; \\
u, v=\text { pesos de produtose insumos; e } \\
r=1 \text { até } \mathrm{m} ; i=1 \text { até } \mathrm{n} ; j=1 \text { até } \mathrm{N}\end{array}$ \\
\hline
\end{tabular}

Já os modelos BCC (VRS) na Tabela 3 admitem a premissa de retorno variável de escala, i.e., qualquer incremento nos insumos pode levar a um acréscimo, não necessariamente proporcional, aos produtos. Esse modelo faz uma avaliação da eficiência técnica (ET) e da eficiência da escala (EE), estimando a eficiência técnica pura, a uma dada 
escala de operações, e identificando se existem ganhos de escala crescentes, decrescentes ou constantes.

Tabela 3. Modelos BCC (VRS) (adaptado de CASA NOVA; SANTOS, 2008)

\begin{tabular}{|c|c|}
\hline $\begin{array}{c}\text { Modelo BCC (VRS) - Orientado ao } \\
\text { Insumo }\end{array}$ & Modelo BCC (VRS) - Orientado ao Produto \\
\hline $\begin{array}{l}\text { Maximizar } \\
\sum_{r=1}^{m} u_{r} * y_{r k}-u_{k}\end{array}$ & $\begin{array}{l}\text { Minimizar } \\
\sum_{i=1}^{n} v_{i} * x_{i k}+v_{k}\end{array}$ \\
\hline $\begin{array}{l}\text { Com as restrições: } \\
\text { 1. } \sum_{r=1}^{m} u_{r} * y_{r j}-\sum_{i=1}^{n} v_{i} * x_{i j}-u_{k} \leq 0 \\
\text { 2. } \sum_{i=1}^{n} v_{i} * X_{i k}=1 \\
\text { 3. } u_{r}, v_{i} \geq 0\end{array}$ & $\begin{array}{l}\text { Com as restrições: } \\
\text { 1. } \sum_{r=1}^{m} u_{r} * y_{r j}-\sum_{i=1}^{n} v_{i} * x_{i j}-v_{k} \leq 0 \\
\text { 2. } \sum_{r=1}^{m} u_{r} * y_{r k}=1 \\
\text { 3. } u_{r}, v_{i} \geq 0\end{array}$ \\
\hline $\begin{array}{l}\text { Sendo: } \\
y_{r}=\text { quantidade de produtor; } \\
x_{i}=\text { quantidade de insumo } i ; \\
u, v=\text { pesos de produtos e insumos; e } \\
r=1 \text { até } \mathrm{m} ; i=1 \text { até } \mathrm{n} ; j=1 \text { até } \mathrm{N}\end{array}$ & $\begin{array}{l}\text { Sendo: } \\
y_{r}=\text { quantidade de produtor } ; \\
x_{i}=\text { quantidade de insumo } i ; \\
u, v=\text { pesos de produtose insumos; e } \\
r=1 \text { até } \mathrm{m} ; i=1 \text { até } \mathrm{n} ; j=1 \text { até } \mathrm{N}\end{array}$ \\
\hline
\end{tabular}

Em respeito às eficiências, pode ser observada a seguinte relação:

Sendo:

$$
E E\left(x_{k}, y_{k}\right)=\frac{E P\left(x_{k}, y_{k}\right)}{E T\left(x_{k}, y_{k}\right)}
$$

$$
\begin{gathered}
E E\left(x_{k}, y_{k}\right)=\text { Eficiência de Escala } \\
\operatorname{EP}\left(x_{k}, y_{k}\right)=\text { Eficiência Produtiva } \\
\operatorname{ET}\left(x_{k}, y_{k}\right)=\text { Eficiência Técnica }
\end{gathered}
$$

Para definir metas globais de redução de consumo de recursos para a holding como um todo, propõe-se um conjunto de dois modelos DEA, todos orientados ao insumo. A opção por dois modelos e não apenas um deve-se ao limitado conjunto de DMU e a percepção de que a redução proporcional de todos os insumos pode não ser factível. Na Tabela 4 apresentam-se as variáveis insumos e produtos em cada dos modelos. Os modelos foram avaliados apenas na formulação VRS, devido a grande heterogeneidade de escala das DMU analisadas.

Tabela 4. Modelos DEA responsáveis pela definição das metas

\begin{tabular}{|c|c|c|}
\hline Modelo & Insumos & Produtos \\
\hline \multirow{2}{*}{1} & Consumo de energia & Valor adicionado distribuído \\
\cline { 3 - 3 } 2 & Consumo de água & Energia gerada \\
\cline { 3 - 3 } & & Valor adicionado distribuído \\
\hline
\end{tabular}

Para a aplicação dos modelos DEA e consequente definição de benchmark, foram levantadas informações dos inputs e outputs, acima mencionados, de acordo com os 
Relatórios de Sustentabilidade de 2017, preenchidos no formato GRI (GRI-G4 ou GRIStandard), de empresas do setor elétrico brasileiro que atuam como geradoras $e$ transmissoras. Ao todo, foram levantadas 12 empresas (incluída a empresa do estudo). Porém, duas empresas tiveram uma qualidade de preenchimento muito abaixo das outras, sendo descartadas para não influenciarem no estudo. Portanto, foram inseridas no modelo nove empresas, mais a holding.

Para desagregar as metas globais entre as unidades de negócio da holding e, consequentemente, compatibilizar as metas individuais com a meta global definida pelo DEA, propõem-se a aplicação do método dos mínimos quadrados, tendo como restrição os limites inferior e superior dos intervalos de previsão para cada mês do horizonte de estudo. No caso de unidades com previsão de crescimento em relação ao ano anterior, a restrição passa a ser a própria previsão, e não o seu limite superior. A formulação matemática do modelo de desagregação das metas globais, obtidas pelo modelo DEA, nas metas locais para as unidades de negócios é apresentada a seguir, em que $M_{i}$ denota a meta (variável de decisão) para a unidade de negócio $i \forall i=1, N$ e $V_{i}$ o respectivo consumo verificado no último ano:

$$
\operatorname{Min} \sum_{i=1}^{N} \frac{1}{V_{i}}\left(M_{i}-V_{i}\right)^{2}
$$

s.a.

$$
\begin{array}{r}
\alpha_{i} \leq M_{i} \leq \beta_{i} \forall i=1, N \\
\sum_{i=1}^{N} M_{i}=\theta V_{\text {Holding }} \\
M_{i} \geq 0 \forall i=1, N
\end{array}
$$

A função objetivo em (2) busca minimizar os desvios entre as metas $M_{i}$ e os respectivos níveis correntes dos consumos $V_{i}$ no último ano. A restrição em (3) restringe as metas $M_{i}$ para o intervalo definido pelos limites do intervalo de previsão [ $\alpha_{\mathrm{i}}, \beta_{\mathrm{i}}$ ] para a i-ésima unidade de negócio. Na restrição (4) $\theta$ é a eficiência da holding determinada pelo modelo DEA e $V_{\text {holding }}$ é o valor verificado do consumo da holding no último ano. Assim, o produto $\theta$ $V_{\text {holding }}$ é a meta global a ser alcançada pela holding. A restrição em (4) garante que a soma das metas locais para as unidades de negócios deve coincidir com a meta global para a holding. Já a restrição (5) é típica dos modelos de otimização e indica a não negatividade das variáveis de decisão.

\section{APLICAÇÃO E RESULTADOS}

A aplicação da metodologia proposta é ilustrada por meio de um estudo de caso com dados provenientes de uma holding com nove unidades de negócios atuantes no setor elétrico. Sem perda de generalidade, no estudo de caso apresentado consideram-se apenas as análises realizadas na definição das metas para dois indicadores: consumo de energia elétrica e consumo de água.

Adicionalmente, considera-se que o horizonte de planejamento do estudo de caso abrange apenas o ano de 2018. Assim, primeiramente foi realizado o levantamento de séries temporais mensais das nove unidades de uma holding no período 2014-2017, portanto, cada série temporal possui 48 observações mensais. Na sequência, o método Loess foi aplicado em todas as séries temporais obtidas com o objetivo de corrigir eventuais outliers presentes nos dados. Em seguida, as séries tratadas foram analisadas pelo programa "NNQ-Estatística" (http://qualimetria.ufsc.br/publicacoes/software/previsao/), desenvolvido pela Universidade Federal de Santa Catarina (UFSC), com o objetivo de identificar o melhor modelo de 
alisamento exponencial para cada série, em cada unidade de negócio. Os melhores modelos para as séries de consumo de água e consumo de energia são apresentados na Tabela 5.

Os modelos ajustados foram utilizados para gerar previsões mensais de janeiro até dezembro de 2018, portanto, um horizonte de previsão de 12 meses. Seguindo a abordagem bottom-up, a soma das previsões mensais para 2018, em cada unidade, resultou na previsão total da holding.

As previsões dos consumos de energia e água para a holding no ano de 2018 são apresentadas na Tabela 6 e Figuras 2 e 3, juntamente como os valores verificados para 2018. Ainda nas Figuras 2 e 3, as linhas tracejadas correspondem ao intervalo de confiança de previsão de 95\%, os pontos os dados reais de 2018 e a reta a previsão para 2018.

\begin{tabular}{|l|c|}
\hline \multirow{2}{*}{ Empresa } & Consumo de Energia \\
\cline { 2 - 2 } & Método \\
\hline Unidade 1 & ANA \\
Unidade 2 & MNA \\
Unidade 3 & ANA \\
Unidade 4 & ANA \\
Unidade 5 & MNA \\
Unidade 6 & ANA \\
Unidade 7 & ANA \\
Unidade 8 & MNA \\
Unidade 9 & ANA \\
\hline
\end{tabular}

(a) Melhores métodos para os indicadores de consumo de energia

\begin{tabular}{|c|c|}
\hline \multirow{2}{*}{ Empresa } & Consumo de Água \\
\cline { 2 - 2 } & Método \\
\hline Unidade 1 & MNM \\
Unidade 2 & MNA \\
Unidade 3 & MNA \\
Unidade 4 & MNA \\
Unidade 5 & MNA \\
Unidade 6 & MNM \\
Unidade 7 & ANA \\
Unidade 8 & MNM \\
Unidade 9 & MAA \\
\hline
\end{tabular}

(b) Melhores métodos para os indicadores de consumo de água

Tabela 5. Métodos de previsões que melhor se ajustaram aos dados de cada unidade

\begin{tabular}{|r|r|r|r|}
\cline { 2 - 4 } \multicolumn{1}{c|}{} & \multicolumn{2}{c|}{ Comparação Mensal 2018: Real x Previsão } \\
\hline em MWh & Real & \multicolumn{1}{c|}{ Previsão } & Variação Mensal \\
\hline jan & 6.399 & 6.429 & $0 \%$ \\
fev & 5.711 & 6.179 & $8 \%$ \\
mar & 6.625 & 6.307 & $-5 \%$ \\
abr & 6.154 & 5.960 & $-3 \%$ \\
mai & 5.878 & 5.504 & $-6 \%$ \\
jun & 5.190 & 5.186 & $0 \%$ \\
jul & 5.248 & 5.166 & $-2 \%$ \\
ago & 5.525 & 5.264 & $-5 \%$ \\
set & 5.528 & 5.325 & $-4 \%$ \\
out & 5.723 & 5.659 & $-1 \%$ \\
nov & 5.736 & 5.782 & $1 \%$ \\
dez & 5.912 & 6.036 & $2 \%$ \\
\hline total anual & 69.630 & 68.797 & $-1 \%$ \\
\hline
\end{tabular}

(a) Consumo de energia

\begin{tabular}{|r|r|r|r|}
\cline { 2 - 4 } \multicolumn{1}{c|}{} & \multicolumn{3}{c|}{ Comparação Mensal 2018: Real x Previsão } \\
\hline em m ${ }^{2}$ & \multicolumn{1}{c|}{ Real } & Previsão & Variação Mensal \\
\hline jan & 44.458 & 47.420 & $7 \%$ \\
fev & 42.318 & 45.126 & $7 \%$ \\
mar & 48.757 & 45.419 & $-7 \%$ \\
abr & 46.360 & 43.959 & $-5 \%$ \\
mai & 44.478 & 41.049 & $-8 \%$ \\
jun & 37.935 & 39.318 & $4 \%$ \\
jul & 33.340 & 40.109 & $20 \%$ \\
ago & 37.193 & 41.494 & $12 \%$ \\
set & 36.037 & 42.515 & $18 \%$ \\
out & 39.184 & 40.544 & $3 \%$ \\
nov & 38.405 & 42.295 & $10 \%$ \\
dez & 38.038 & 42.287 & $11 \%$ \\
\hline total anual & 486.503 & 511.535 & $5 \%$ \\
\hline
\end{tabular}

(b) Consumo de água

Tabela 6. Valores mensais previstos e verificados dos consumos de energia e de água 


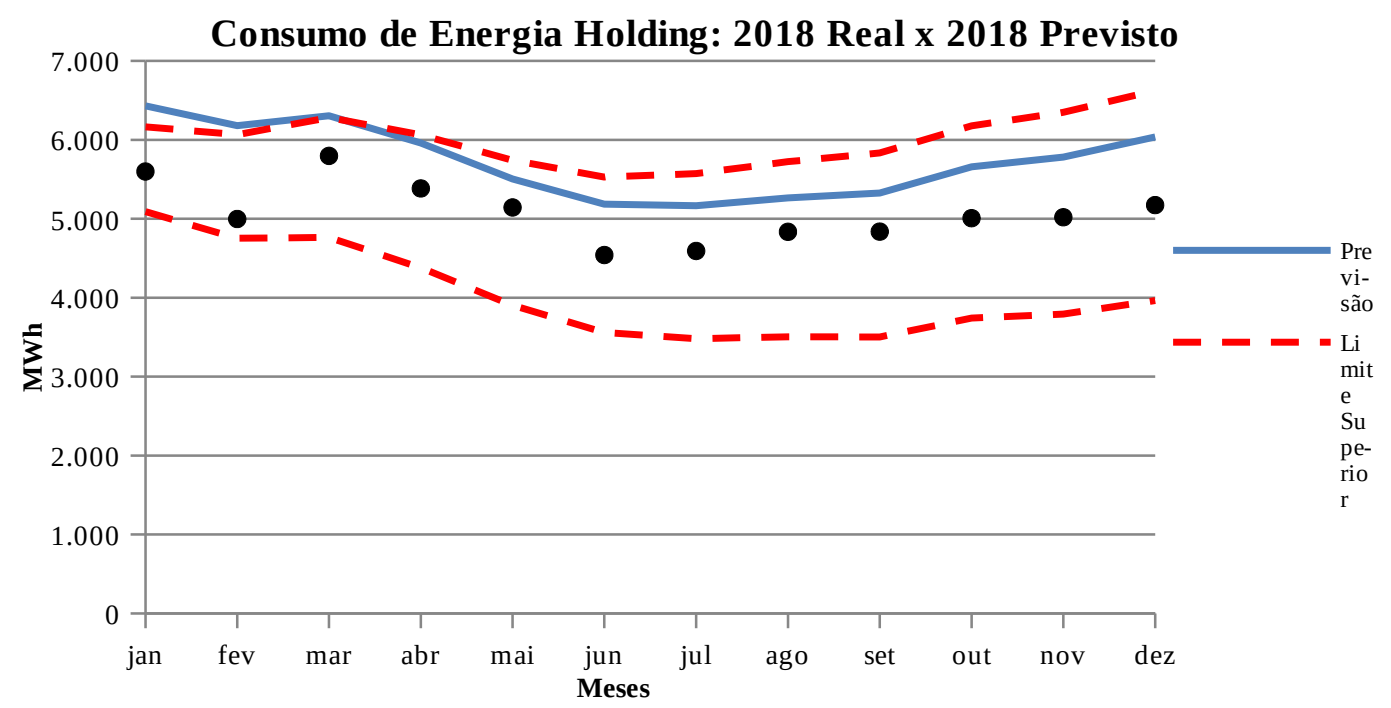

Figura 2. Comparação entre o consumo de energia previsto e real em 2018

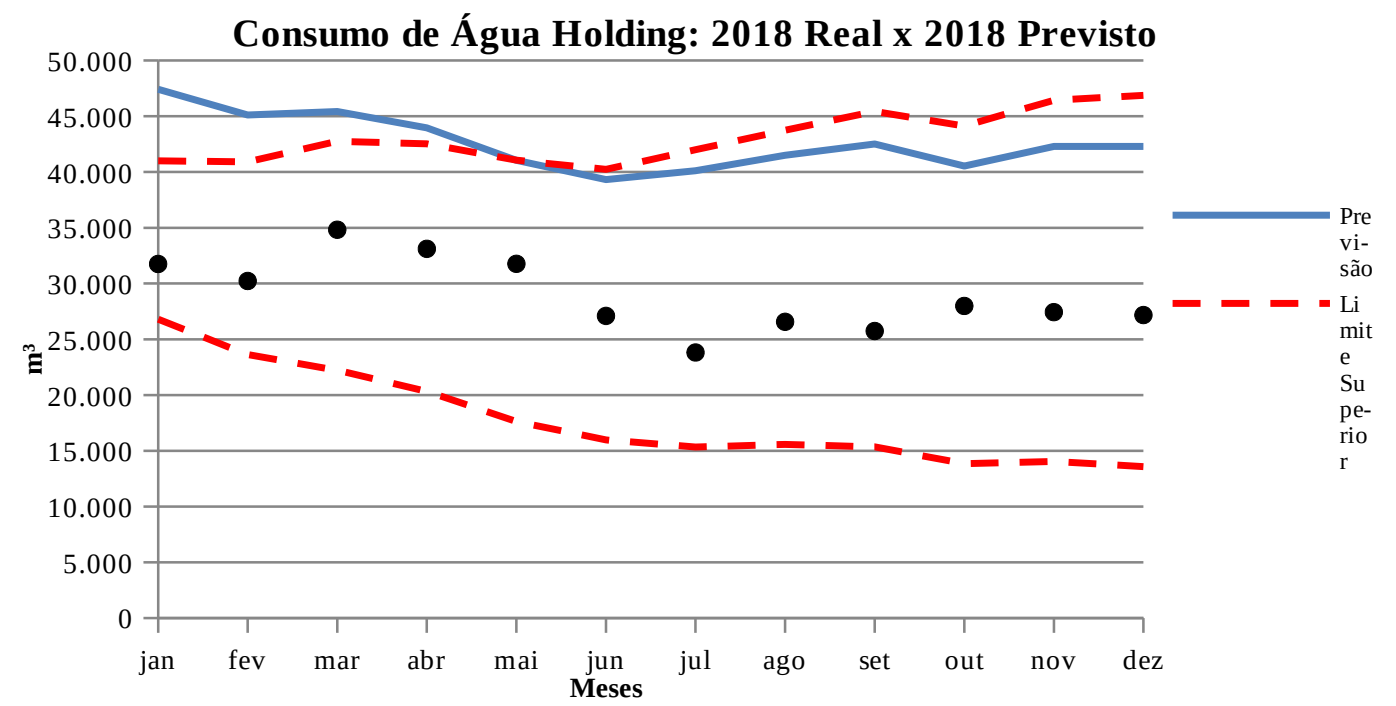

Figura 3. Comparação entre o consumo de água previsto e real em 2018

\subsection{Metas para o Consumo de Energia}

Os resultados dos modelos DEA (VRS, orientado ao insumo), com o consumo de energia como único insumo, são apresentados na Tabela 7 abaixo. Observa-se que a holding é eficiente no modelo VRS. Portanto, no modelo VRS, a holding é o próprio benchmarking. 
Tabela 7. Eficiências VRS em relação ao consumo de energia (Modelo 1)

\begin{tabular}{|l|r|}
\hline \multicolumn{1}{|c|}{ Empresa } & Eficiência VRS \\
\hline Empresa & \\
1 & 0,001617675 \\
Holding & \\
Empresa & \\
2 & \\
Empresa & 0,23284908 \\
3 & 0,006364428 \\
Empresa & \\
4 \\
Empresa & 0,567805187 \\
5 & 0,454286347 \\
Empresa & \\
6 & 1 \\
Empresa & \\
7 \\
Empresa \\
8 \\
Empresa \\
9
\end{tabular}

Como a holding já é eficiente, não há um percentual de redução como meta. Então, será definida uma meta global de manutenção, ou seja, manter o consumo de energia igual ao ano anterior. O resultado se encontra na Tabela 8 abaixo.

Tabela 8. Distribuição da meta de manutenção para o consumo de energia

\begin{tabular}{|c|c|c|c|c|c|c|}
\hline Unidades & $\begin{array}{l}\text { Consumo Real } \\
2017 \text { (MWh) }\end{array}$ & $\begin{array}{c}\text { Previsão } \\
2018 \text { (MWh) }\end{array}$ & $\begin{array}{l}\text { Meta } 2018 \text { Dis- } \\
\text { tribuída (MWh) }\end{array}$ & $\begin{array}{c}\text { Meta \% } \\
(2017-2018)\end{array}$ & $\begin{array}{l}\text { Consumo Real } \\
2018 \text { (MWh) }\end{array}$ & $\begin{array}{c}\text { Meta seria } \\
\text { atingida? }\end{array}$ \\
\hline $\begin{array}{r}\text { Unidade } \\
1\end{array}$ & 1.026 & 538 & 965 & $-6,0 \%$ & 517 & Sim \\
\hline Unidade & & & & & & \\
\hline $\begin{array}{r}2 \\
\text { Unidade }\end{array}$ & 1.149 & 1.107 & 1.150 & $0,1 \%$ & 1.025 & Sim \\
\hline & 3.732 & 3.642 & 3.735 & $0,1 \%$ & 4.611 & Não \\
\hline & 3.332 & 3.722 & 3.335 & $0,1 \%$ & 3.217 & Sim \\
\hline & 5.553 & 5.355 & 5.558 & $0,1 \%$ & 5.571 & Não \\
\hline $\begin{array}{r}6 \\
\text { Unidade }\end{array}$ & 6.822 & 6.296 & 6.828 & $0,1 \%$ & 6.284 & Sim \\
\hline $\begin{array}{r}7 \\
\text { Unidade }\end{array}$ & 7.008 & 6.890 & 7.014 & $0,1 \%$ & 6.691 & Sim \\
\hline $\begin{array}{r}8 \\
\text { Unidade }\end{array}$ & 16.939 & 16.008 & 16.954 & $0,1 \%$ & 16.248 & Sim \\
\hline 9 & 26.593 & 25.239 & 26.616 & $0,1 \%$ & 25.167 & Sim \\
\hline Holding & 72.154 & 68.797 & 72.154 & $0,0 \%$ & 69.331 & Sim \\
\hline
\end{tabular}

\subsection{Metas Para o Consumo de Água}

Os resultados do modelo DEA (VRS, orientado ao consumo) para o input de consumo de água estão presentes na Tabela 9 a seguir. Observa-se que, novamente, a holding é eficiente no modelo VRS. Portanto, no modelo VRS, a holding é o próprio benchmarking. 
Tabela 9. Eficiências VRS em relação ao consumo de água (Modelo 2)

\begin{tabular}{|l|r|}
\hline \multicolumn{1}{|c|}{ Empresa } & Eficiência VRS \\
\hline Empresa & \\
1 & 0,035049959 \\
Holding & 1 \\
Empresa & \\
2 & 0,000318208 \\
Empresa & \\
3 & 0,007425765 \\
Empresa & 0,000797606 \\
4 & 1 \\
Empresa & \\
5 & 1 \\
Empresa & \\
6 & \\
Empresa & \\
7 & \\
Empresa & \\
8 & 0,430790507 \\
Empresa & \\
9 & 0,526713245 \\
\hline
\end{tabular}

Como no caso a holding já é eficiente, não há um percentual de redução como meta. Então, será definida uma meta global de manutenção, ou seja, manter o consumo de água igual ao ano anterior. $\mathrm{O}$ resultado da desagregação da meta global da holding entre as suas unidades, pelo método de mínimos quadrados, se encontra na Tabela 10 abaixo.

Tabela 10. Distribuição da meta de manutenção para o consumo de água

\begin{tabular}{|c|c|c|c|c|c|c|}
\hline Unidades & $\begin{array}{l}\text { Consumo Real } \\
2017\left(\mathrm{~m}^{3}\right)\end{array}$ & $\begin{array}{l}\text { Previsão } \\
2018\left(\mathrm{~m}^{3}\right)\end{array}$ & $\begin{array}{l}\text { Meta } 2018 \text { Distri- } \\
\text { buída }\left(\mathrm{m}^{3}\right)\end{array}$ & $\begin{array}{c}\text { Meta \% } \\
(2017-2018)\end{array}$ & $\begin{array}{l}\text { Consumo Real } \\
2018\left(\mathrm{~m}^{3}\right)\end{array}$ & $\begin{array}{c}\text { Meta seria } \\
\text { atingida? }\end{array}$ \\
\hline Unidade & & & & & & \\
\hline $\begin{array}{r}1 \\
\text { Unidade }\end{array}$ & 1.577 & 959 & 1.804 & $14,4 \%$ & 3.080 & Não \\
\hline & 50.932 & 40.933 & 55.432 & $8,8 \%$ & 55.623 & Não \\
\hline $\begin{array}{r}\text { Unidade } \\
3\end{array}$ & 23.528 & 21.782 & 24.938 & $6,0 \%$ & 27.604 & Não \\
\hline Unidade & & & & & & \\
\hline $\begin{array}{r}4 \\
\text { Unidade }\end{array}$ & 112.920 & 129.586 & 111.685 & $-1,1 \%$ & 113.335 & Não \\
\hline $\begin{array}{r}5 \\
\text { Unidade }\end{array}$ & 33.404 & 29.582 & 34.157 & $2,3 \%$ & 27.073 & Sim \\
\hline $\begin{array}{r}6 \\
\text { Unidade }\end{array}$ & 38.693 & 40.980 & 44.522 & $15,1 \%$ & 45.602 & Não \\
\hline $\begin{array}{r}7 \\
\text { Unidade }\end{array}$ & 30.562 & 28.621 & 32.888 & $7,6 \%$ & 42.253 & Não \\
\hline $\begin{array}{r}8 \\
\text { Unidade }\end{array}$ & 178.840 & 208.375 & 164.421 & $-8,1 \%$ & 160.351 & Sim \\
\hline$\underline{9}$ & 11.703 & 10.717 & 12.312 & $5,2 \%$ & 11.240 & Sim \\
\hline Holding & 482.159 & 511.535 & 482.159 & $0,0 \%$ & 486.161 & Não \\
\hline
\end{tabular}




\section{CONCLUSÕES}

Conclui-se que a definição de metas de sustentabilidade a partir de previsões mensais calculadas pelos métodos de alisamento exponencial, considerando o intervalo de confiança identificado a partir da análise histórica de séries temporais de indicadores, pode auxiliar as empresas no alcance dos ODS identificados como prioritários. A definição do percentual de variação da meta em relação ao intervalo de confiança pode variar de acordo com o interesse, a política da empresa ou com sua capacidade de definir planos de ação mais ou menos desafiadores em relação aos temas tratados, como por exemplo, em relação à redução no consumo de energia, água e geração de resíduos. O uso de um benchmarking também pode ser um apoio para definição de metas, combinando o objetivo de atingir a eficiência do mercado com o intervalo de confiança de previsão da empresa.

Nota-se que é sempre importante a identificação de outliers, não só utilizando métodos de tratamento de dados, como o Loess, por exemplo, mas também com a realização de análise qualitativa da série temporal com a finalidade de identificar erros de preenchimento ou situação e eventos não fortuitos.

Assim, observa-se que a definição de metas de sustentabilidade utilizando métodos de previsão como os de alisamento exponencial, associados à análise de discrepância de dados, análise da fonte da série de dados, identificação de outliers e definição de benchmarking, podem garantir definições de metas desafiadoras mais adequadas à realidade das empresas do setor, considerando o contexto dos países onde possuem suas atividades de geração, transmissão ou distribuição. Além disso, este tipo de metodologia pode tornar o processo de definição de metas algo dinâmico, parte integrante da gestão de sustentabilidade, mas que precisa ser constantemente avaliado e melhorado para melhor apoiar as decisões gerenciais estratégicas. Algumas sugestões de melhorias são o uso de outros métodos de previsão e utilizar o DEA para comparar a holding com empresas internacionais.

Portanto, a metodologia proposta é promissora e pode ser adaptada para ser aplicada em outras áreas além da sustentabilidade e por outras empresas, não apenas para o setor elétrico.

\section{REFERÊNCIAS BIBLIOGRÁFICAS}

[1] BERTOLO, Luiz Antonio. Métodos básicos de previsão no Excel. Catanduva-SP, 2013. Disponível em:

http://www.bertolo.pro.br/MetodosQuantitativos/Simulacao/MetodosBasicosDePrevisa oDeSeriesTemporaisNoExcel.pdf. Acesso em: 5 out. 2018.Primeira referência. Primeira referência. Primeira referência.

[2] CASA NOVA, Silvia Pereira de Castro; SANTOS, Ariovaldo. Aplicação da análise por envoltória de dados utilizando variáveis contábeis. Revista de Contabilidade e

Organizações, São Paulo, 2008. Disponível em:

http://www.revistas.usp.br/rco/article/view/34717. Acesso em: 23 mar. 2019.

[3] CEBDS, 2018. Guia de ODS para empresas. Disponível em:

https://cebds.org/publicacoes/guia-dos-ods/\#.XPBHG4hKjIU. Acesso em: 29 mar. 2019.

[4] COSTA, Sayonara Fernandes; BOENTE, Diego Rodrigues. Avaliação da eficiência econômico-financeira das empresas integrantes do índice de sustentabilidade empresarial por meio da análise envoltória de dados. Revista Ambiente Contábil, Natal, 2011. Disponível em: https://periodicos.ufrn.br/ambiente/article/view/1358. Acesso em: 21 mar. 2019. 
[5] MAKRIDAKIS, Spyros; SPILIOTIS, Evangelos; ASSIMAKOPOULOS, Vassilios. Statistical and Machine Learning forecasting methods: Concerns and ways forward. PLOS ONE, [S. l.], 2018. Disponível em: https://journals.plos.org/plosone/article/file? id=10.1371/journal.pone.0194889\&type=printable. Acesso em: 21 ago. 2019.

[6] MARQUETTI, Adalmir; VIALI, Lori. Princípios e aplicações de regressão local. Análise Econômica, Porto Alegre, v. 22, n. 42, p. 253-277, 2004. Disponível em: https://seer.ufrgs.br/AnaliseEconomica/article/view/10808/6418. Acesso em: 16 abr. 2019.

[7] PESSANHA, José Francisco Moreira et al. Implementando modelos DEA no R. In: SIMPÓSIO DE EXCELÊNCIA EM GESTÃO E TECNOLOGIA, 2013, Rio de Janeiro. Anais [...]. Rio de Janeiro: Associação Educacional Dom Bosco, 2013. Disponível em: https://www.aedb.br/seget/arquivos/artigos13/44218525.pdf. Acesso em: 21 mar. 2019.

[8] R CORE TEAM. R: A language and environment for statistical computing. R Foundation for Statistical Computing, Vienna, Áustria, 2019. Disponível em: https://www.R-project.org/. Acesso em: 5 fev. 2019

[9] RESENDE, Marcelo. Relative efficiency measurement and prospects for yardstick competition in brazilian electricity distribution. Energy Policy, [S. l.], 2002. Disponível em: https://www.sciencedirect.com/science/article/pii/S030142150100132X. Acesso em: 28 mar. 2019.

[10] SARTORI, Simone. Proposta de método de avaliação integrada de sustentabilidade com uso da análise envoltória de dados. 2016. 251 p. Tese (Doutorado em Engenharia de Produção) - Universidade Federal de Santa Catarina, Florianópolis, 2016. Disponível em: https://repositorio.ufsc.br/xmlui/handle/123456789/168099. Acesso em: 15 mar. 2019.

[11] SOUZA, Matheus Alves Madeira; RODRIGUES, Lásara Fabrícia; FARIA, Gilson Ataliba. Análise envoltória de dados aplicada ao setor elétrico de transmissão brasileiro. In: SIMPÓSIO BRASILEIRO DE PESQUISA OPERACIONAL, 2016, Vitória. Anais [...]. [S. 1.]: Sociedade Brasileira de Pesquisa Operacional, 2016. Disponível em: http://www.din.uem.br/sbpo/sbpo2016/pdf/155893.pdf. Acesso em: 29 mar. 2019.

[12] UFSC. NNQ - Estatística. Florianópolis, 2008. Disponível em: http://qualimetria.ufsc.br/publicacoes/software/previsao/. Acesso em: 23 jan. 2019.

[13] VERÍSSIMO, Andrey Jonas et al. Métodos estatísticos de suavização exponencial HoltWinters para previsão de demanda em uma empresa do setor metal mecânico. Revista Gestão Industrial, Paraná, v. 8, n. 4, p. 154-171, 2012. DOI 10.3895/S1808044820120004. Disponível em: https://periodicos.utfpr.edu.br/revistagi/article/view/1378. Acesso em: 4 out. 2018.

[14] ZHOU, Haibo et al. Data envelopment analysis application in sustainability: The origins, development and future directions. European Journal of Operational Research, [S. 1.], v. 264, 2017. Disponível em: https://www.sciencedirect.com/science/article/abs/pii/S0377221717305623. Acesso em: 28 mar. 2019. 